\title{
RENAL HEMANGIOMA
}

\author{
THEODORICO F. DA COSTA NETO, JUAN M. RENTERIA, G. DI BIASE FILHO
}

\author{
Section of Urology, Ipanema General Hospital, Rio de Janeiro, RJ, Brazil
}

\begin{abstract}
Introduction: Renal hemangioma is a relatively rare benign tumor, seldom diagnosed as a cause of hematuria.

Case report: A female 40-year old patient presented with continuous gross hematuria, anemia and episodic right lumbar pain, with onset about 3 months previously. The patient underwent multiple blood transfusions during her hospital stay and extensive imaging propedeutics was performed. Semirigid ureterorenoscopy evidenced a bleeding focus in the upper calix of the right kidney, with endoscopic treatment being unfeasible.

The patient underwent right upper pole nephrectomy and presented a favorable outcome. Histopathological analysis of the surgical specimen showed that it was a renal hemangioma.

Comments: Imaging methods usually employed for diagnostic investigation of hematuria do not have good sensitivity for renal hemangioma. However, they are important to exclude the most frequent differential diagnoses. The ureterorenoscopy is the diagnostic method of choice and endoscopic treatment can be feasible when the lesion is accessible and electrocautery or laser are available. We emphasize the open surgical treatment as a therapeutic option upon failure of less invasive methods.
\end{abstract}

Key words: kidney; hemangioma; hematuria

Int Braz J Urol. 2004; 30: 216-8

\section{INTRODUCTION}

Renal hemangioma is a relatively rare benign tumor, seldom diagnosed as a cause of hematuria. Approximately 200 cases were reported since this condition was first described by Virchow in 1867 (1). This tumor can be classified as cavernous or capillary and the majority of lesions have a diameter of less than $1 \mathrm{~cm}$. It affects most frequently young adults and there is no preference for gender.

Clinical presentation is characterized by hematuria ranging from intermittent microscopic hemorrhage to abundant, continuous bleeding, with hemodynamic repercussion.

We report one case of gross hematuria due to renal hemangioma emphasizing clinical presentation, imaging diagnosis and the treatment prescribed.

\section{CASE REPORT}

Female, 40-year old patient, complained of episodic gross hematuria with onset around 4 years previously, which became continuous and associated with episodic right lumbar pain in the past 3 months.

On the physical examination, gross hematuria and anemia were evident. The patient underwent an ultrasonography of the urinary tract, excretory urography and abdominal and pelvic computerized tomography that did not evidence significant alterations. The semi-rigid ureterorenoscopy showed a bleeding focus in the upper calix of the right kidney, with endoscopic treatment being impossible due to difficulty of access. Renal arteriography was performed, showing no alteration (Figure-1). The patient maintained active bleeding, leading to persistent anemia and re- 


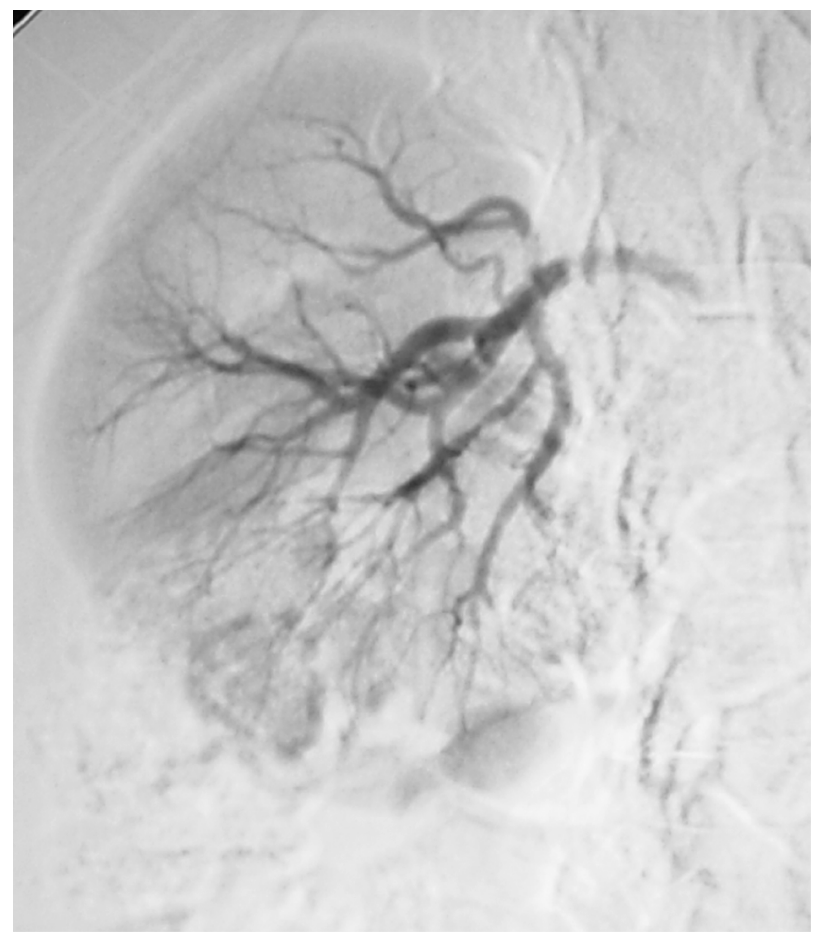

Figure 1 - Right selective renal arteriography, without identifying the bleeding focus.

quiring multiple blood transfusions during hospitalization. Renal arteriography was unable to evidence the bleeding focus. However, considering its previous location on semi-rigid ureterorenoscopy, an option was made towards selective embolization of the right kidney upper pole (Figure-2).

Since there was no hematuria remission following arterial embolization, the patient underwent a right upper pole nephrectomy (Figure-3), presenting a favorable outcome, without hematuria within a 6month follow-up. Histopathological analysis revealed renal hemangioma.

\section{COMMENTS}

Renal hemangioma is seldom diagnosed as a cause of hematuria. Imaging examinations usually are not helpful for diagnosing hemangioma, though they are important in order to exclude more frequent causes of hematuria.

The differential diagnosis of renal hemangioma must include papillary necrosis, ectopic pa-

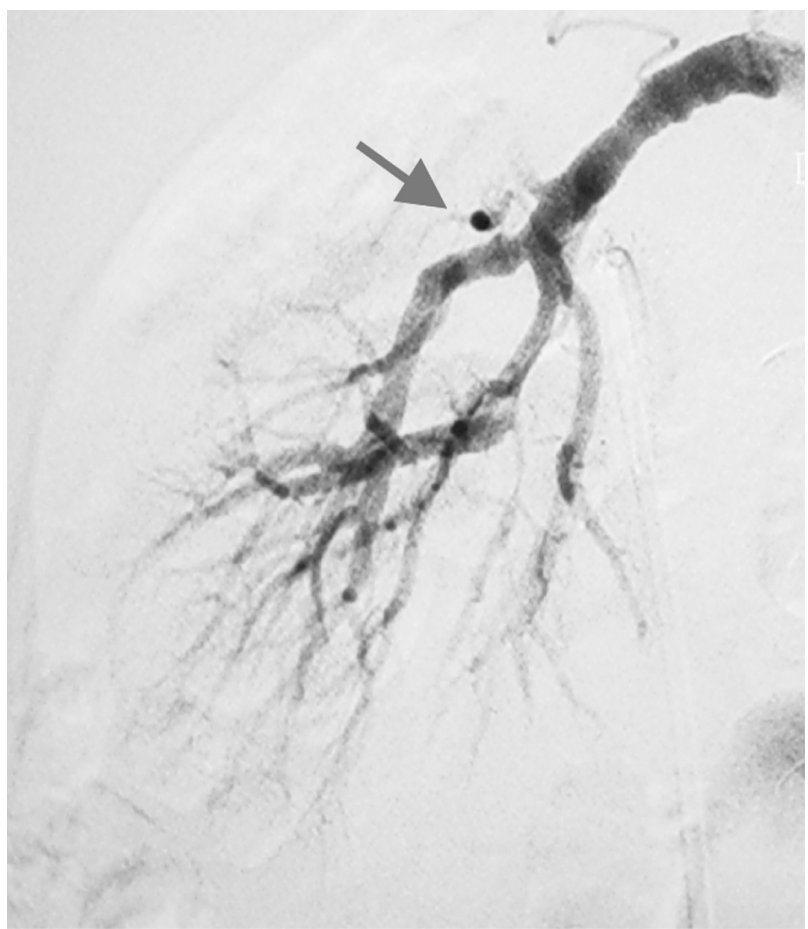

Figure 2 - Right renal arteriography following selective embolization of the upper pole (arrow).

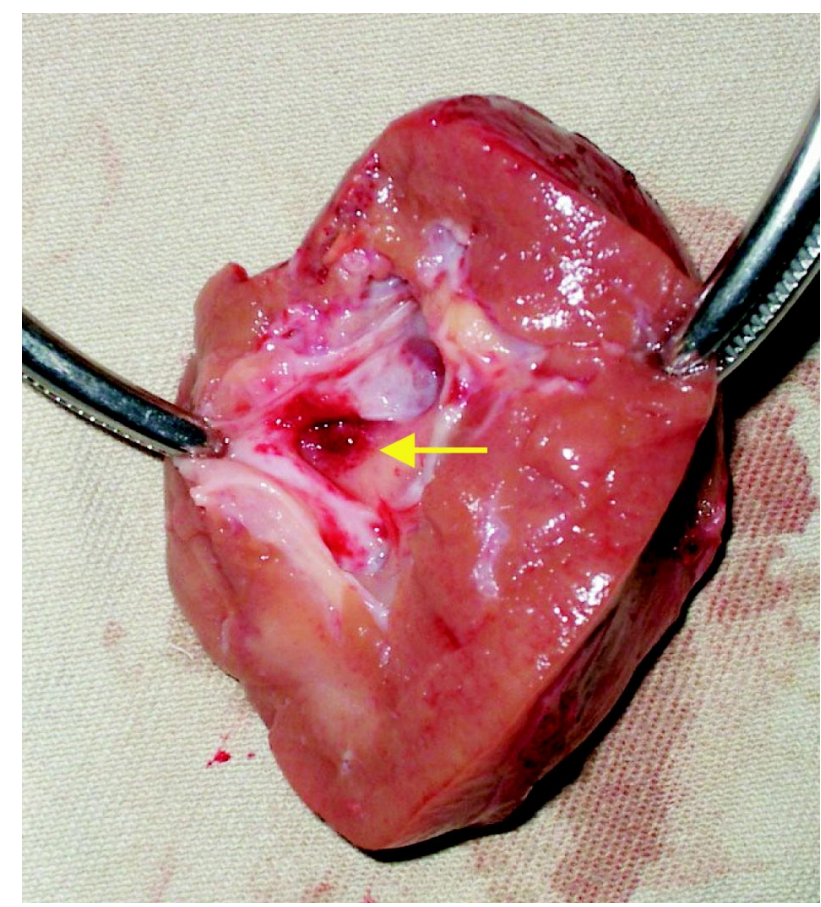

Figure 3 - The macroscopic surgical specimen revealed a lesion measuring approximately $0.5 \mathrm{~cm}$ in the upper calix of the right kidney (arrow). 
pilla, hemorrhagic papillitis and urothelial carcinoma, among others (2).

Currently, the flexible ureterorenoscopy is the method of choice for diagnosing this kind of lesion (3). When available, the electrocautery or laser constitutes also the treatment of choice, if the lesion is accessible. However, in the absence of such facilities, open surgery is indicated in cases of persistent bleeding.

\section{REFERENCES}

1. Daneshmand S, Huffman JL: Endoscopic management of renal hemangioma. J Urol. 2002; 167: 488-9.

2. Viguier JL, Abbar M, Gelet A, Bouvier R, Martin X, Marechal JM, et al.: The contribution of endoscopy in the diagnosis of unilateral hematuria of renal origin and pseudotumors of the upper urinary tract. Prog Urol. 1994; 4: 219-27.

3. Tawfiek ER, Bagley DH: Ureteroscopic evaluation and treatment of chronic unilateral hematuria. J Urol. 1998; 160: 700-2.
Received: November 25, 2003 Accepted after revision: January 28, 2004

\section{Correspondence address:}

Dr. Juan Miguel Renteria

Hospital Geral de Ipanema

Rua Antônio Parreiras, 67 / 69

Rio de Janeiro, RJ, 22411-020, Brazil

Fax: + $55213111-2362$

E-mail:jm_renteria@yahoo.com.br 